\title{
Praktische Implikationen und Bausteine moderner Interessenvermittlung
}

Das nachfolgende Kapitel stellt praktische Implikationen für die moderne Interessenvermittlung vor, die sich als Verknüpfung aus den Ergebnissen der empirischen Analyse und den theoretischen Überlegungen ableiten. Die verschiedenen Aspekte sind zu Bausteinen aggregiert, die jeweils die gesamte Bandbreite der aufgezeigten Handlungslogiken innerhalb eines Aspekts darstellen. Die Bausteine ergeben nur in der Zusammenschau Sinn. Denn viele Aspekte der einzelnen Bausteine bedingen sich gegenseitig und dürfen nicht isoliert betrachtet werden. Insgesamt konnten sechs Bausteine der modernen Interessenvermittlung analytisch herausgearbeitet werden (siehe Abb. 6.1). Während die Bausteine 1, 2 und 3 eng mit den theoretischen Grundlagen des Dreiecks der Handlungsrationalität verbunden sind, lösen sich die Bausteine 4, 5 und 6 stärker von den theoretischen Bezugspunkten. Dies folgt zum einen aus dem Einbezug der Kontextfaktoren und zum anderen aus einer veränderten, übergeordneten Perspektive.

\section{Strategische Repräsentation}

Der Baustein der strategischen Repräsentation thematisiert die gewandelte Sichtweise, wie Organisationen die politische Interessenvertretung von fluiden und punktuellen Interessen handhaben. Darin wird die idealtypische Vorstellung aufgelöst, dass eine Organisation eine kontinuierliche, basisdemokratisch legitimierte Interessenvertretung darstellen muss und einer festen und homogenen Unterstützer*innenschaft bedarf. In der politischen Praxis zeigen die veränderten Unterstützungsstrukturen einen Trend zur strategischen, fluiden Kooperation zwischen Unterstützer*innen, Organisation und politischen Entscheidungsträger*innen. Diese veränderten Bedingungen erzeugen vielfältige politische Möglichkeiten. Gleichzeitig erodieren sichergeglaubte Strukturen und Bündnisse allmählich. 


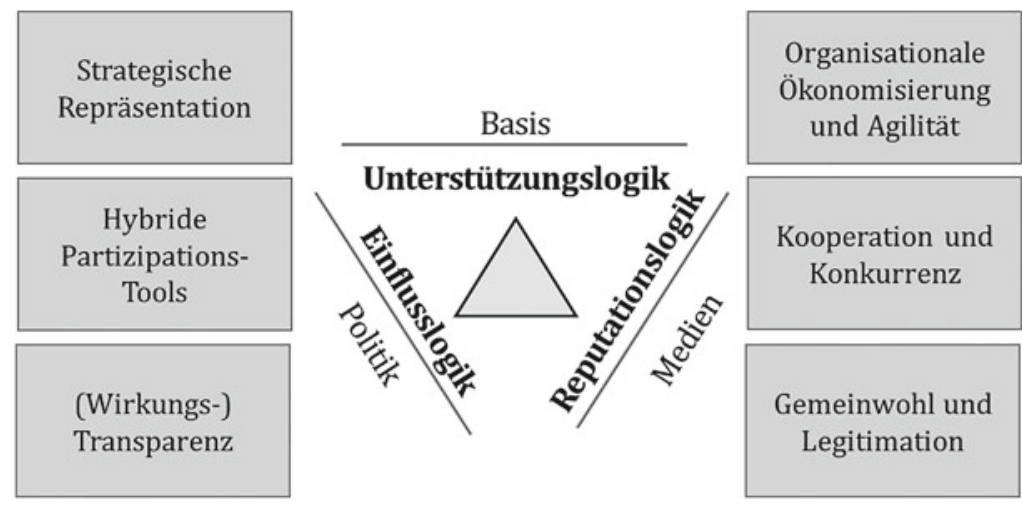

(Grafik: eigene Darstellung)

Abbildung 6.1 Sechs Bausteine der modernen Interessenvermittlung

Dieser Trend stützt sich auf die empirische Beobachtung, dass Organisationen vermehrt diskutieren, ob eine feste Mitgliedschaft mit basisdemokratischen Strukturen das beste Mittel der Wahl ist, um - vergleichbar mit Verbänden - ihre aggregierten Interessen gegenüber den politischen Entscheidungsträger*innen vorzubringen. Im Datenmaterial zeigt sich die Loslösung vom verbandlichen Ideal hin zu fluiden Strukturen. Darunter fällt die Möglichkeit für Unterstützer*innen, sich beispielsweise nur situativ, d. h. etwa bei einer einzelnen Aktion zu beteiligen. Strukturell schlagen sich diese punktuellen Formen des Engagements in Fördermitgliedschaften mit regelmäßigen Beitragszahlungen, jedoch ohne vereinsrechtliche Mitsprachemöglichkeiten nieder. So erweitert sich die klassische Interessenvertretung um die strategische Repräsentation.

Die Entwicklung von inhaltlichen Forderungen und die Unterstützungsmobilisierung können so getrennt voneinander stattfinden. Je nach Position oder Kampagne kann eine Gruppe unterschiedliche Unterstützer*innen werben. Die mobilisierbaren Interessen variieren über die bisherigen Gruppengrenzen hinweg. Vielmehr setzen sich die zu vertretenden Gruppen kurzfristig und kontextabhängig zusammen. Ausgewählte Themen der Mitglieder, aber auch eines breiteren Unterstützer*innenkreises werden aufgenommen. Auf diese Weise wird die Organisation nicht nur für die eigene Mitgliedschaft, sondern auch für die diffusen Unterstützer*innenkreise interessant.

Diese Auflösungs- und Differenzierungserscheinungen berühren die Kernüberlegung der neo-korporatistischen Austauschbeziehung, d. h. die Vertretung demokratisch aggregierter Interessen einer festen Gruppe und deren Mobilisierungsfähigkeit sowie Compliance der Mitglieder als Tauschgut. Die Angebote, 
die eine Organisation an ihre Unterstützer*innen macht, differenzieren sich aus. Eine direkte Interessenaggregation und -vertretung bei gleichzeitiger Kontrolle über die Organisationsspitze muss nicht mehr ein Kernangebot von NGOs sein.

Organisationsentscheidungen werden aufgrund von Komplexität und der Notwendigkeit von Organisationswissen zunehmend in die Geschäftsstelle verlagert. Die basisdemokratische Kontrolle erfolgt nur in geringem Maß über vereinsrechtliche Instrumente wie jährliche Mitgliederversammlungen, sondern indirekt über die Unterstützungsbekundung für ein Thema, beispielsweise durch die Unterzeichnung einer Petition, die Teilnahme an einer Aktion, oder die Beibehaltung der regelmäßigen finanziellen Zuwendung. Die Organisationen erreichen dadurch mehr Menschen über den Kreis ihrer Mitglieder hinaus. Dies vergrößert den Hebel, um politischen Druck auf Entscheider*innen und Medien auszuüben. In den Vordergrund rücken die öffentliche Sichtbarkeit und aktionsbasierte Formate. Klassische, vereinsrechtliche Strukturen treten zurück.

Dieser Trend verändert die Austauschbeziehung zu politischen Entscheidungsträger*innen. Wenn fluide Unterstützungsgruppen vermehrt und eher punktuell mit Entscheidungsträger*innen in Kontakt treten, können politische Kompromisse nicht in gleicher Form wie mit aggregierten Interessenorganisationen getroffen werden. Anders als bei den langfristig gewachsenen Vertrauensverhältnissen zwischen etablierten Verbänden und der Politik muss die Frage, wer mit welcher gesellschaftlichen Teilgruppe verhandelt, situationsabhängig ausgehandelt werden. Der Unterschied zeigt sich deutlich, wenn das wertvolle Tauschgut der Mitglieder- bzw. Unterstützer*innen-Compliance wegfällt. Stark aggregierte, teils von breiten Bündnissen getragene Kampagnen schaffen allerdings neue Opportunitätsstrukturen für die Politik - besonders, wenn populäre Entscheidungen zu Gunsten dieser Bündnisse zur „low hanging fruit" für die eigene Profilierung werden (siehe Abb. 6.2).

\footnotetext{
Praxisimplikation: Der Schulterschluss zwischen Entscheidungsträger*innen und den größeren mobilisierten Kreisen gewinnt punktuell an Bedeutung bei Themen mit hoher politischer und medialer Relevanz. Als neuer Austauschmodus trägt die strategische Repräsentation dazu bei, mit einer veränderten Ausgangslage umzugehen. So ist erkennbar, dass neue Modi des Austausches gefunden werden - wie die strategische Repräsentation und deren Akzeptanz - um auf heterogene, diffuse und nicht langfristig organisierbare Interessen eingehen zu können.
}

Abbildung 6.2 Praxisimplikation für strategische Repräsentation 


\section{Hybride Partizipationstools}

Der Baustein der hybriden Partizipationstools erfasst das neuausdifferenzierte Spektrum an Partizipationsmöglichkeiten, welches Interessenorganisationen einsetzen. Diese Möglichkeiten reichen von klassischem ehrenamtlichen Engagement auf lokaler Ebene, über kontinuierliche Mitgliedschaft bis hin zu punktuellem Online-Protest (Click-Aktivismus). Grundlegend ist die empirische Beobachtung der veränderten Mitgliederstruktur und der Beteiligungserwartungen aus dem Unterstützungskreis. Daher steht dieser Baustein im engen Bezug zur strategischen Repräsentation.

Idealtypisch ist die Vorstellung einer basisdemokratisch aufgebauten Organisation, deren Mitglieder die Möglichkeit des lokalen Engagements und der direkten Mitbestimmung haben. Die Arbeit und der Fortbestand dieser Organisationen ist primär davon bestimmt, dass ehrenamtliche Funktionspositionen besetzt sind und die innerorganisationale Demokratie ausgefüllt wird. Empirisch zu beobachten ist die Auflösung und Ausdifferenzierung dieses idealtypischen Modells. So breitet sich ein ganzes Spektrum an Partizipationstools in der Organisationspraxis aus. Mitglieder und Unterstützer*innen können wählen zwischen festen, losen oder punktuellen Unterstützungsmöglichkeiten, zwischen digitaler Beteiligung oder Präsenz. Auch bei ihren finanziellen oder zeitlichen Ressourcen steht ihnen offen, sich aktiv einzubringen, ehrenamtlich zu arbeiten sowie bestimmte Anliegen über einmalige, projektbezogene Spenden oder regelmäßige Förderbeiträge zu finanzieren. Die individuelle Entscheidung, kein Mitglied zu sein, wirkt nicht ausschließend, da es sowieso keine klassischen Mitglieder mehr gibt.

Die modernen Organisationen, welche sich in den vergangenen zwei Jahrzehnten gegründet haben - mit Greenpeace als großem Vorreiter in diesem Bereich - haben ihre Organisationsstrukturen verstärkt auf dieses Modell des optin optout Engagements ausgerichtet und zielen bei langfristiger Verbundenheit eher auf finanzielle Unterstützung. Das neue Geschäftsmodell kann zunächst als Reaktion auf zurückgehende Zahlen im klassischen Engagement gewertet werden, deckt sich aber auch mit den Anforderungen der starken inhaltlichen Spezialisierung, Kompetenzbündelung und Zentralisierung der Organisationen. Hinzu kommen die Sorge vor gezielter Unterwanderung der Mitgliedschaft, die die Handlungsfähigkeit einschränkt, sowie der erhebliche finanzielle Aufwand, den der Aufbau und die Pflege ehrenamtlicher Strukturen bedeuten würde (siehe LobbyControl und Foodwatch Abschnitt 5.1). Die nachträgliche Gestaltung ehrenamtlicher Strukturen ist hingegen eher mit normativen Überlegungen hinsichtlich des Aufbaus und Erhalts innerorganisationaler Demokratiestrukturen verbunden (siehe LobbyControl Abschnitt 5.1). Gleiches gilt für die grundsätzliche basisdemokratische Ausrichtung (siehe Attac Abschnitt 5.1). 
Weiterhin kann beobachtet werden, dass die klassischen basisdemokratisch aufgebauten Organisationen ihre etablierten Beteiligungsformen anreichern und ihr Angebot diversifizieren. Es entstehen hybride Formen, die zwischen punktueller und kontinuierlicher Unterstützung liegen und digitale wie analoge Welten in Verbindung bringen. Digitale Kommunikation und Mobilisierung können analogen Bewegungen neue Impulse verschaffen. Die Aggregation und Vertretung von Interessen erhalten damit eine neue Qualität, da sehr genau nachzuvollziehen ist, welches Thema wie viel (kurzfristige) Unterstützung erfährt. Öffentliche Aktionen und mediale Sichtbarkeit als ein Angebots- und Nachfragepaar in der Austauschbeziehung zwischen Organisationen und Unterstützer*innen, gewinnen enorm an Bedeutung. Vieles folgt der Medienlogik, um die öffentliche Sichtbarkeit, thematische Salienz und Popularität zu erhöhen und damit der Outside-Lobbying-Strategie mehr Kraft zu geben (siehe Abb. 6.3).

\footnotetext{
Praxisimplikation: Angepasste Partizipationsangebote für eine ausdifferenzierte Nachfrage der Basis zu schaffen, ist zentral für das politische Überleben und erfolgreiches Handeln von Organisationen. Für mitgliedschaftsbasierte Organisationen heißt dies, Unterstützungsmöglichkeiten per digitaler Abstimmung und Deliberation einzusetzen. Aufgrund der begrenzten Aufnahmefähigkeit der politischen Agenda kann ein Überangebot an Partizipationsmöglichkeiten für Mitglieder und Interessierte allerdings ineffizient werden. Bei inflationärem Einsatz gehen Petitionen, E-Mailaktionen und punktuelle Kampagnen in der Konkurrenz um Aufmerksamkeit unter.
}

Abbildung 6.3 Praxisimplikation für hybride Partizipationstools

\section{Transparenz und (Selbst-)Wirksamkeit}

Der dritte Baustein der Transparenz und (Selbst-)Wirksamkeit greift die geänderten Präferenzen der Unterstützer*innen zivilgesellschaftlicher Organisationen auf, die zunehmend den Wunsch äußern, genau nachvollziehen zu können, welche Auswirkung das eigene Engagement tatsächlich hat. Diese Forderung schließt an die sich wandelnden Mitgliedschafts- und Beteiligungsmöglichkeiten an, die die Organisationen ihren Unterstützer*innen anbieten. 
Das klassische Organisationsangebot, dass die Mitglieder über binnendemokratische Strukturen die Führungsspitze kontrollieren können, ist je nach spezifischer Organisationsform unterschiedlich ausgeprägt. Insbesondere bei Organisationen, die vom Verbandsvorbild abweichen, ist diese Kontrolle weniger auf direktem Wege als über indirekte Formen der Zustimmung und Kritik vorhanden.

Der Wunsch der Basis, die Arbeit der Organisationsspitze zu kontrollieren, wird im hohen Maße abgelöst durch die Forderung nach Überprüfbarkeit und Nachweisbarkeit der Organisationswirkung als Selbstwirksamkeitserfahrung der Unterstützer*innen. Einher geht damit die Forderung nach mehr Transparenz über innerorganisationale Strukturen und ganz explizit über die Verwendung von Mitteln sowie die Forderung nach einer transparenten Darstellung der erreichten Erfolge. Unterstützer*innen wollen nachvollziehen können, wie sich ihr finanzielles oder zeitliches Engagement tatsächlich auswirkt. Hieran wird eine Verlagerung von der Ergebnis- zur Wirkungsorientierung deutlich (siehe Abschnitt 5.7).

Von vielen Seiten wurde allerdings deutlich gemacht, dass Transparenz nicht als Selbstzweck verfolgt werden sollte. Die herausgegebenen Organisationsdaten müssen auch einen greifbaren Informationsgehalt aufweisen, denn Glaubwürdigkeit stellt ein hohes Gut für Organisationen dar. Die nachvollziehbare Darstellung von sichtbaren Erfolgen erhält damit nicht nur eine neue Funktion, sondern auch eine neue Qualität (siehe Abb. 6.4).

\footnotetext{
Praxisimplikation: Den gewachsenen Ansprüchen der Mitglieder und Unterstützer*innen muss geschicktes Erwartungsmanagement gegenübergestellt werden. Trotz der neuen Transparenzstandards und der finanziellen, ehrenamtlichen und punktuellen Unterstützungsmöglichkeiten, bleiben Politikwandelprozesse komplex, zeitintensiv und langwierig. Große politische Erfolge sind äußerst selten; einzelne Unterschriften und Aktionsteilnahmen reichen für gesellschaftliche Veränderung nicht aus. Organisationen müssen eine Übersetzungsleistung erbringen, um ein Bewusstsein für neue politische Realitäten zu schaffen.
}

Abbildung 6.4 Praxisimplikation für Transparenz und (Selbst-)Wirksamkeit

\section{Organisationale Ökonomisierung und Agilität}

Der Baustein organisationale Ökonomisierung und Agilität richtet sich an die strategischen Überlegungen von Organisationen, um auf die Anforderungen von Basis, Medien und Politik adäquat zu reagieren. Als Reaktion auf die zunehmende 
Komplexität der Aufgaben lässt sich eine fachliche Professionalisierung des Personals ausmachen. Die Mitarbeiter*innen (Non-Profit-Manager*innen, Campaigner*innen, Fundraiser*innen, Social-Media-Agent*innen und andere) werden über ein wachsendes Angebot von Studiengängen und Weiterbildungen ausgebildet und zertifiziert. In den Geschäftsstellen sind die Arbeitsabläufe effizient gestaltet, arbeitsteilig formalisiert und an aktuelle betriebswirtschaftliche Maßgaben angepasst. Die professionalisierte Kampagnen- und Medienarbeit wird kombiniert mit einer professionell konzipierten Außenwirkung: Der Markenkern und der unique selling point werden über Beraterstrukturen herausgearbeitet und verfeinert - bis die Organisation eine eigenständige Marke ist.

Bei gelungener Professionalisierung können Organisationen agil und flexibel arbeiten, ohne in unkoordinierten und tagespolitischen Aktionismus zu verfallen. Die Geschwindigkeit und die Schlagzahl, in der Themen verarbeitet und gesetzt werden, haben sich stark erhöht und an die digital-mediale Logik angepasst. In ständiger Konkurrenz um öffentliche Aufmerksamkeit ist eine professionelle Medienarbeit überlebenswichtig.

Auch bei Organisationen, die sich nicht der professionalisierten Kampagnenarbeit verschrieben haben, lassen sich spürbare Veränderungen ausmachen. Stellvertretend sind hier Organisationen zu nennen, die u. a. lokalen Naturschutz betreiben. Eine professionalisierte Darstellung der lokalen Erfolge und professionelle Mitgliederbindung hat auch in den meisten Ortsgruppen Einzug gehalten, wenngleich in unterschiedlicher Qualität.

Trotz der organisationalen Professionalisierung, die insgesamt viele Vorteile bietet, bleibt eine zentrale Einschränkung bestehen: Die zunehmende Komplexität der Themen erzwingt eine starke fachliche Spezialisierung oder eine Übersetzungsleistung, die die relevanten Inhalte vereinfacht. Daher kommt es zu einer Abwägung zwischen inhaltlicher Arbeit und öffentlichkeitswirksam vermittelbaren Bildern. Bei aller Professionalität und Planung kann ein zufälliges externes Ereignis immer noch dafür sorgen, dass eine Kampagne keine Sichtbarkeit erfährt oder ein Thema unvorhergesehen an enormer Dynamik gewinnt. Beispielsweise wurde das Thema der Rodung des Hambacher Forsts von unterschiedlichen Gruppen seit Jahren bearbeitet und dynamisierte sich 2018 für viele Beteiligte überraschend stark. Nicht nur die Kampagnenarbeit ist professionalisiert, sondern auch das Einwerben von Geldern durch Fundraising und Projektfinanzierung. Auch hier ist zu erkennen, dass die Organisationen auf Veränderungstrends der Spendenlandschaft bereits reagieren und weitere Entwicklungen antizipieren (siehe Abb. 6.5). 
Praxisimplikation: Gerade die gewachsene, organisationale Professionalisierung der Interessenvermittlung unterstreicht die Notwendigkeit, eine Rückbindung an den Unterstützungskreis aufrechtzuerhalten bzw. zu beleben. Die Rückkopplung zur Basis nimmt Zeit in Anspruch, was mit der gesteigerten Schlagzahl der Themenverarbeitung vereinbart werden muss. Organisationen müssen die zeitliche Möglichkeit für NichtEntscheidungen und Nicht-Positionierung innerhalb und außerhalb ihrer Kernthemen schaffen.

Abbildung 6.5 Praxisimplikation für organisationale Ökonomisierung und Agilität

\section{Kooperation und Konkurrenz}

Der Baustein Kooperation und Konkurrenz stellt die zivilgesellschaftliche Arbeitsteilung und die Spezialisierung des eigenen Profils in der interessenlandschaftlichen Nische in den Mittelpunkt. Die empirischen Ergebnisse verdeutlichen die Tendenz zu einer kooperativen Grundorientierung, die die Organisationen gemeinschaftlich verfolgen.

Auch den Austausch- und Kooperationsbeziehungen unter den Organisationen liegen schwierige strategische Überlegungen zugrunde. Dass es Konkurrenz um Aufmerksamkeit, mediale Sichtbarkeit, Unterstützer*innen und Spenden gibt, ist selbstverständlich. Folglich reagieren die professionalisierten Organisationen darauf, indem sie die eigene ökologische Nische (siehe Abschnitt 5.4) in der Interessenlandschaft identifizieren und auszufüllen versuchen. Eine Arbeitsteilung und Spezialisierung der Zivilgesellschaft ist seit Jahren zu beobachten. Trotz - oder gerade wegen - der Konkurrenzsituation auf dem zivilgesellschaftlichen Markt ist der temporäre Zusammenschluss zu Kampagnenbündnissen oder Themenallianzen ein beliebtes, aber zweischneidiges Strategiemittel.

Als empirischer Kristallisationspunkt diente dieser Studie das Anti-TTIPBündnis, das sinnbildlich für gelungene Kooperation und die Möglichkeit des Überkommens von Organisationsunterschieden steht. Schwierig blieb aber auch bei diesem erfolgreichen Bündnis die klare Zuordnung von Siegen und Erfolgen. Für teilnehmende Organisationen besteht immer die Gefahr, dass die KostenNutzen-Rechnung für sie individuell nicht positiv ausfällt, weil sich das finanzielle und personelle Engagement weder vorteilhaft bei den Spendeneinnahmen noch bei der Mitgliederzahl niederschlägt (siehe Abb. 6.6). 
Praxisimplikation: Bündnisse und Themenallianzen sind für Organisationen ein wichtiges strategisches Mittel. Doch auch wenn inhaltliche Grundüberzeugung oder breite Forderungsposition häufig übereinstimmen, sollten Organisationen ihre Kooperation selektiv wählen und sich nur dort beteiligen, wo eine wirkliche Profilierung über die Anerkennung des Themas oder eines Organisationserfolgs möglich ist.

Abbildung 6.6 Praxisimplikation für Kooperation und Konkurrenz

\section{Gemeinwohl und Legitimation}

Der sechste Baustein des Gemeinwohls und der Legitimation greift die Diskussion über eine Gemeinwohlorientierung von Organisationen im Prozess der Interessenvermittlung auf. Diese letzte Perspektive soll mit einem prozeduralen Gemeinwohlverständnis zur Versachlichung der Debatte beitragen.

Im gegenwärtigen breiten Konsens der politikwissenschaftlichen Debatte zum prozeduralen Gemeinwohl wird davon ausgegangen, dass es in modernen, pluralistisch demokratischen Gesellschaften keinen vordefinierten Begriff des Gemeinwohls geben kann und geben darf (vgl. Strünck 2014; siehe Abschnitt 5.7). Der Vorstellung eines a priori gegebenen Gemeinwohls, welches von politischen Akteuren gleichsam intuitiv erkannt werden kann, wird eine klare Absage erteilt. Demokratisches Gemeinwohl muss vielmehr prozedural geschaffen, d. h. durch den Ausgleich unterschiedlicher, gesellschaftlicher Interessen auf Grundlage eines fairen Prozesses erzeugt und so immer wieder neu ermittelt werden (Schuppert 2002: 24, Lietzmann 2002: 298, Strünck 2014). Erst am Ende des demokratischen Prozesses steht das Gemeinwohl a posteriori auf der gesellschaftlichen Makroebene.

Dieser Verhandlungsprozess hat sich in verfassungsrechtlich festgelegten Verfahren graduell immer weiter geöffnet - die Einbeziehung der Verbände im Korporatismus war gesellschaftlichen Veränderungen und einer nötigen Erweiterung geschuldet (Lietzmann 2002: 300; 301). Auch NGOs und andere zivilgesellschaftliche Akteure haben sukzessive in diesem Prozess eine aktive Rolle erlangt. So fasste Hans Lietzmann bereits 2002 die immer noch aktuelle Diskussion um die Legitimation neuer Gemeinwohlakteure - so auch NGOs - zusammen:

„Der Blick zurück zeigt, dass auf jeder Stufe der Erweiterung der Kreise der Gemeinwohlakteure nach der Berechtigung und der politischen Legitimität der Newcomer in der politischen Entscheidungsarena gefragt wurde“ (Lietzmann 2002: 301). 
Unter den befragten Interviewpartner*innen finden sich die verschiedenen Aspekte wieder. Die eigene Gemeinwohlorientierung einer Organisation wird dabei auch als strategisches Legitimationsargument verwendet. Sie dient dazu, sich gegenüber ökonomisch motivierten Partikularinteressen abzugrenzen oder dem Gegenüber fehlende Gemeinwohlorientierung vorzuwerfen. Die Gemeinwohlorientierung steht aber auch sinnbildlich für übergeordnete Themen, die die gesamte Gesellschaft in weitgefasstem Verständnis betreffen. Darunter fallen u. a. die bereits genannten Menschenrechte sowie Naturschutz- oder Globalisierungsthemen. So können Argumentationsmuster und Selbstverständnis moralischnormativ aufgeladenen werden - ohne jedoch in das kritisierte Feld einer Gemeinwohldefinition a priori zu gelangen. Darüber hinaus ist zu beobachten, dass im Bereich der Interessenvertretung und des politischen Willensbildungsprozesses der Bezug auf Gemeinwohl als Vorwurf und als Selbstüberhöhung verwendet wird, um so Positionen von Konkurrenzorganisationen zu entkräften. Der Gemeinwohlbezug verkommt in dieser Argumentationsstrategie zum sinnentleerten Kampfbegriff.

Eine weniger verkrampfte Diskussion mit einer Verständigung darauf, dass es in der deutschen Demokratie nur einen prozeduralen, verfassungsrechtlich abgesicherten Gemeinwohlbegriff gibt, könnte die Vorwürfe und überhöhte Selbstzuschreibungen entkräften. Keine Organisation, ob wirtschaftlichen oder öffentlichen Interessen verpflichtet, muss eine Argumentation über einen individuellen, inhaltsbestimmten Gemeinwohlanspruch hinnehmen. Zu akzeptieren ist aber, dass die Aufnahme von NGOs am Aushandlungsprozess um Gemeinwohl eine neue Realität ist, weil sie zu wichtigen Stakeholdern im Politikgestaltungsprozess geworden sind (siehe Abb. 6.7).

\footnotetext{
Praxisimplikation: In der politischen Debatte muss der Bezug auf Gemeinwohl zurückhaltend und vorsichtig erfolgen. Der Konsens des prozeduralen Gemeinwohls verdeutlicht, dass zahlreiche Argumente mit Gemeinwohlbezügen lediglich strategisch verwendet werden. Dies beschränkt die Aussagekraft sowohl von Gemeinwohl als Legitimationsargument, als auch des instrumentellen Vorwurfs, dem politischen Gegner die Gemeinwohlorientierung abzuerkennen. Der gesamtgesellschaftliche Aushandlungsprozess ist und bleibt die Richtschnur.
}

Abbildung 6.7 Praxisimplikation für Gemeinwohl und Legitimation 
Open Access Dieses Kapitel wird unter der Creative Commons Namensnennung 4.0 International Lizenz (http://creativecommons.org/licenses/by/4.0/deed.de) veröffentlicht, welche die Nutzung, Vervielfältigung, Bearbeitung, Verbreitung und Wiedergabe in jeglichem Medium und Format erlaubt, sofern Sie den/die ursprünglichen Autor(en) und die Quelle ordnungsgemäß nennen, einen Link zur Creative Commons Lizenz beifügen und angeben, ob Änderungen vorgenommen wurden.

Die in diesem Kapitel enthaltenen Bilder und sonstiges Drittmaterial unterliegen ebenfalls der genannten Creative Commons Lizenz, sofern sich aus der Abbildungslegende nichts anderes ergibt. Sofern das betreffende Material nicht unter der genannten Creative Commons Lizenz steht und die betreffende Handlung nicht nach gesetzlichen Vorschriften erlaubt ist, ist für die oben aufgeführten Weiterverwendungen des Materials die Einwilligung des jeweiligen Rechteinhabers einzuholen. 